\title{
Poststroke seizure: optimising its management
}

\author{
Michael $Y X u$
}

To cite: Xu MY. Poststroke seizure: optimising its management. Stroke and Vascular Neurology 2019;4: e000175. doi:10.1136/svn2018-000175

Received 23 June 2018 Revised 12 September 2018 Accepted 11 October 2018 Published Online First 9 December 2018

\section{Check for updates}

(c) Author(s) (or their employer(s)) 2019. Re-use permitted under CC BY-NC. No commercial re-use. See rights and permissions. Published by BMJ.

Department of Neurology, OSF Illinois Neurological Institute, University of Illinois College of Medicine, Peoria, Illinois, USA

Correspondence to Dr Michael Y Xu; Michael.Y.Xu@INI.ORG

\section{ABSTRACT}

Seizure after stroke or poststroke seizure (PSS) is a common and very important complication of stroke. It can be divided into early seizure and late seizure, depending on seizure onset time after the stroke. It has been reported that ischaemic and haemorrhagic stroke accounts for about $11 \%$ of all adult epilepsy cases and $45 \%$ of epilepsy cases over 60 years of age. However, there are no reliable guidelines in clinical practice regarding most of the fundamental issues of PSS management. In recent years there has been an increased interest in the study of PSS which may give clinical practitioners a better picture of how to optimise PSS management. Studies have indicated two peaks in PSS occurrence - the first day and 6-12 months after a stroke. Haemorrhagic stroke, cortical involvement, severity of initial neurological deficit, younger patients ( $<65$ years of age), family history of seizures and certain genetic factors carry a higher risk of PSS. The use of continuous electroencephalogram has demonstrated significant benefits in capturing interictal or ictal abnormalities, especially in cases of non-convulsive seizures and non-convulsive status epilepticus. Current available data indicated that there was no significant difference in antiepileptic efficacy among most of the antiepileptic drugs (AEDs) in PSS. Levetiracetam and lamotrigine are the most studied newer generation AEDs and have the best drug tolerance. The purpose of this review is to summarise the recent advances in PSS research and focus on the most important practice issues of PSS management.

\section{INTRODUCTION}

According to the Centres for Disease Control and Prevention (CDC), stroke is the fifth leading cause of death in the USA and is a major cause of serious disability in adults. ${ }^{1}$ In the past decade, advances in stroke treatment have dramatically reduced the mortality rate of stroke. Meanwhile, the number of stroke survivors with significant complications and disability has increased. ${ }^{2}$ Seizure is an important complication after stroke. It has been reported that ischaemic and haemorrhagic stroke accounts for about $11 \%$ of all adult epilepsy cases and $45 \%$ of epilepsy cases over 60 years of age. ${ }^{3}$ However, improvement of poststroke seizure (PSS) management has not evolved as fast as other aspects of stroke management. So far, there are no reliable clinical practice guidelines regarding most of the fundamental issues of PSS management. ${ }^{4-7}$
Neurologists and other clinical practitioners are faced with uncertainties in their daily care of patients with PSS, more so confounding information continues to challenge medical care providers. For example: (1) How to make a correct diagnosis of PSS? (2) Should we treat every patient with PSS and when should we start treatment? (3) What is an appropriate antiepileptic drug (AED) for PSS? The aim of this review is to summarise the recent advances in PSS research and focus on important practice issues of PSS diagnosis and management.

\section{CLASSIFICATION}

PSS can be classified into early-onset and lateonset seizures because their underlying pathological mechanisms and the risk of developing into epilepsy are different. Studies have shown that early-onset PSS may result from acute neuron injury and subsequent glutamate-mediated excitotoxicity, ion channel dysfunction and blood barrier disruption. ${ }^{4-10}$ In contrast, the mechanisms behind late-onset PSS may be secondary gliotic scarring with associated changes in membrane properties, chronic inflammation, neurodegeneration, altered synaptic plasticity, eventually leading to hyperexcitability, and increased synchronisation of neuronal activities. ${ }^{11}{ }^{12}$ However, the time cut-off for early versus late seizures is arbitrary and varies among different studies. Early seizure (ES) has been defined as 24-48 hours, 1 week, 2 weeks or 30 days poststroke presentation. ${ }^{13-17}$ Seizures occurring at least 1-2 weeks poststroke presentation are called late seizure (LS). ${ }^{715-17}$

In studies involving seizures after stroke, PSS and poststroke epilepsy (PSE) are the most commonly used terms. Although the terminology seems interchangeable among several studies, according to their definition and based on the underlying pathology, they represent two different clinical conditions. The commonly used definition of epilepsy has been defined as two or more unprovoked seizures occurring greater than 24 hours apart. But a new definition, which was introduced in 
2014, allows a diagnosis of epilepsy only after one seizure, if the probability of further seizures is similar to the general recurrence risk (at least 60\%), after two unprovoked seizures, occurring over the next 10 years. ${ }^{18}$ The above statement blurred the meaning of the definition of seizure and epilepsy to a certain degree, especially in the case of seizures after stroke, or other conditions with the unclear probability of subsequent seizures. As a result, some studies involving seizures after stroke consider later onset seizures as PSE, as supported by data showing high recurrence risk (55\%-90\%); however, the recurrence risk is not consistent among all studies. ${ }^{19-21}$ Hence, some studies have disregarded these definitions and only consider PSE as two unprovoked seizures ( $>24$ hours apart) occurring after a stroke. ${ }^{41322}$

\section{PREVALENCE, RISK FACTORS AND OUTCOME}

Because of the differences in the definitions of ES and LS being used also, disparities in study design, patient cohort and stroke subtype (ischaemic or haemorrhagic), the incidence of ES has been reported as 2.2\%-33\%, while that of LS varies from $3 \%$ to $67 \% .{ }^{7131516}$ In spite of all these variances, the incidence pattern is consistent. There are two peaks in PSS occurrence-the first usually occurs within the first day after a stroke, and the second between 6 and 12 months, respectively. ${ }^{71315}$

Many studies have examined the risk factors associated with PSS, and showed that haemorrhagic stroke, cortical involvement, severity of initial neurological deficit (high NIH Stroke Scale/Score (NIHSS)), younger patients (<65years of age), family history of seizures and genetic factors (rs671, CD41-1 and so on) carry higher risk of PSS or PSE. ${ }^{4} 1416172223$ However, the strength of association between PSS and risk factors is variable and differs across studies. Several studies tried to develop a measurement tool to predict the risk for PSE. ${ }^{51416172223}$ In a prospective study, Strzelczyk et al ${ }^{24}$ evaluated 264 consecutive patients with stroke, and defined seven risk items, weighted differently (table 1), then established the Post-Stroke Epilepsy Risk Scale. The scale showed moderate sensitivity (70\%) and positive predictive value $(87.5 \%)$, and relatively high specificity $(99.6 \%)$ and negative predictive value

\begin{tabular}{ll}
\hline $\begin{array}{l}\text { Table } 1 \\
\text { Scale }\end{array}$ & Seven items of the Post-Stroke Epilepsy Risk \\
\hline Item & Weight \\
\hline $\begin{array}{l}\text { Supratentorial stroke } \\
\text { ICH involving cortical areas }\end{array}$ & 2 \\
\hline $\begin{array}{l}\text { Ischaemia involving cortical or cortical-subcortical } \\
\text { areas }\end{array}$ & 1 \\
\hline Ischaemia + ongoing neurological deficit & 1 \\
\hline $\begin{array}{l}\text { Stroke caused neurological deficit with mRS }>3 \\
\text { Seizure occurred up to 14 days after stroke }\end{array}$ & 1 \\
\hline Seizure occurred 15 days or later after stroke & 2 \\
\hline
\end{tabular}

$\mathrm{ICH}$, intracerebral haemorrhage; mRS, modified Rankin scale.

\begin{tabular}{ll}
\hline Table 2 CAVE score (for LS from ICH) \\
\hline CAVE & Risk of LS \\
\hline C: cortical involvement (1 point) & 0 point: $0.6 \%$ \\
A: age $<65$ years (1 point) & 1 point: $3.6 \%$ \\
V: volume $>10 \mathrm{~mL}$ (1 point) & 2 points: $9.8 \%$ \\
E: early seizure (1 point) & 3 points: $34.8 \%$ \\
& 4 points: $46.2 \%$ \\
\hline
\end{tabular}

$\mathrm{ICH}$, intracerebral haemorrhage; LS, late seizure.

(98.8\%). Haapaniemi et $a 2^{25}$ completed a retrospective analysis of intracerebral haemorrhage (ICH) cases and developed the CAVE (cortical involvement, age, volume, early seizure) score for predicting LS occurrence after ICH (table 2). The risk of LS was $0.6 \%, 3.6 \%, 9.8 \%$, $34.8 \%$ and $46.2 \%$ for CAVE scores $0-4$, respectively. The c-statistics were $0.81(0.76-0.86)$ and $0.69(0.59-0.78)$ in the validation cohort. But more studies are required to confirm the values for these measurement tools.

Regarding the effects of PSS on mortality and functional outcome of stroke survivors, animal studies suggest that frequent ES in acute ischaemic stroke can increase infarct size and impair functional recovery. This is triggered by increased metabolic and oxygen demands on ischaemic tissues surrounding the infarcted area, as a result of seizures. Most clinical studies have found that PSS is associated with poor poststroke functional recovery and outcome. ${ }^{519222627}$ According to Xu et al $\mathrm{s}^{28}$ meta-analysis on postischaemic stroke seizures (PISS) and stroke outcome, the overall mortality rates for patients with and without PISS were $34 \%$ (95\% CI 27\% to $42 \%$ ) and $18 \%$ (95\% CI $12 \%$ to $23 \%$ ), respectively. The overall prevalence rate of disability in patients with and without PISS was $60 \%(95 \%$ CI $32 \%$ to $87 \%)$ and $41 \%(95 \%$ CI $25 \%$ to $57 \%$ ), respectively. In a subgroup analysis, the authors also found that mortality and disability were significantly elevated as a result of early-onset seizures. A recent community-based sample study by Stefanidou et al reported PSS was significantly associated with moderate (HR 4.33) and severe (HR 9.71) disability. ${ }^{13}$ Studies also found that seizures immediately following stroke are associated with increasing resources utilisation and prolonged length of hospital stay. ${ }^{132029}{ }^{30}$ Furthermore, PSS in older patients has a negative impact on quality of the life through driving restrictions, increased risk of falls and fractures, and increased susceptibility to adverse effects from the use of AEDs. ${ }^{13} 29-33$ However, conflicting results exist. A large study failed to identify PSS as an independent risk factor of 2-year mortality, ${ }^{52}$ and similar results have been reported by other investigators. ${ }^{34}$ Alberti $e t a l^{35}$ reported ES does not seem to be associated with adverse outcome at hospital discharge after acute stroke.

\section{CLINICAL MANIFESTATIONS AND DIAGNOSIS OF PSS}

According to the 2017 International League Against Epilepsy (ILAE) classification of seizures, ${ }^{36}$ PSS, like 
other seizures with different aetiologies, can present as focal onset seizures, with or without impaired awareness (old terms of simple or complex partial seizures), with or without motor symptoms, focal onset seizure with extension of bilateral tonic-clonic seizures (old term of secondary generalised tonic-clonic seizures). Some PSS can also present as generalised onset or unknown onset. Stefanidou $e \mathrm{al}^{13}$ in their community-based cohort study found $72 \%$ (18 of 25) of PSS were focal onset seizures, among them 22\% (4 of 18) with evolution on bilateral convulsion. Twenty-eight per cent (7 of 25) of PSS were generalised onset seizures. In a population-based longterm comprehensive follow-up study, Bryndziar et al ${ }^{19}$ found that $66 \%$ of PSS were focal (partial) onset seizures, with or without secondary generalisation. Thirty-four per cent of PSS were generalised onset seizures, and $11.4 \%$ of PSS developed into status epilepticus (SE).

The recognition and diagnosis of PSS, however, have not always been an easy task. In fact, most PSS occurred in patients who already had significant neurological deficits, so the new symptoms originated by seizures may be hidden or ignored. ${ }^{737}$ In certain instances, PSS present as only subtle clinical findings, such as focal, intermittent eye deviation or nystagmus, mild facial twitching, or focal sensation changes; other PSS cases may only have worsening of motor deficit, impaired speech or fluctuating stroke recovery. Moreover, patients with PSS may have no focal motor or sensory signs, and only present with altered mental status and behaviour arrest. These cases of subclinical/non-convulsion seizures or SE can only be confirmed by electroencephalogram (EEG) ${ }^{38-40}$ Claassen et $a l^{38}$ in their study of continuous EEG (cEEG) monitoring in intensive care unit (ICU) patients reviewed 570 consecutive patients who underwent cEEG, among them $37 \%$ were stroke-related (19\% subarachnoid haemorrhage, $7.9 \% \mathrm{ICH}$ and $9.8 \%$ ischaemic stroke). The authors found that seizures were detected in $19 \%$ of patients who underwent cEEG monitoring; the seizures were exclusively non-convulsive in $92 \%$ of these patients. According to the definition proposed in this study, seizures were considered convulsive if any of the following was described: 'generalized tonic-clonic seizures', 'grand mal seizures', 'convulsions', 'rhythmic jerking', 'rhythmic twitching' or similar descriptions. If none of these was present and cEEG confirmed seizures, the seizures were considered non-convulsive, whether or not subtle movements (eg, subtle facial twitching, eye deviation, nystagmus) were observed. Miyaji et a $t^{41}$ recently reported 127 patients with PSS, $29 \%$ of them were non-convulsive seizures. Among them, 60 patients (47\%) developed into SE, including 11 patients $(9 \%)$ without convulsion.

In addition, various non-seizure transients, for example, hypokinetic and hyperkinetic movement, convulsive movement, and intermittent transient neurological deficit (eg, transient ischaemic attack (TIA)), can further complicate the diagnosis of PSS. ${ }^{42}{ }^{43}$ Seizurelike involuntary movements related to specific stroke subtypes have also been reported. Several studies and serial case reports described vertebrobasilar occlusive and brainstem stroke with convulsive-like movement. ${ }^{44}$ It has been hypothesised that these movements are related to ischaemia of the corticospinal tract rather than a true seizure. ${ }^{44}$ Stroke involving the basal ganglia or its pathways and the frontal lobe has been reported, causing hemichorea and hemiballismus. ${ }^{42}{ }^{44} \mathrm{Kim}^{45}$ also described nine patients with anterior cerebral artery stroke presenting with hemiparkinsonism or asterixis, which may be misdiagnosed as focal seizures. TIA with limb shaking has been described as a particular type of seizure-like movement thought to be secondary to focal cerebral hypoperfusion due to severe stenotic or occlusive contralateral carotid disease. ${ }^{11}$ Clues which may help distinguish limb-shaking TIAs from seizures are lack of a Jacksonian march, no epileptiform discharges or electrographic seizures on EEG, ineffectiveness of AEDs, precipitation of symptoms with manoeuvres that cause cerebral hypoperfusion, and cessation of symptoms when improving cerebral perfusion. Manoeuvres such as rising from a chair, hyperventilation and hyperextension of the neck may provoke shaking movements, whereas lying supine may eliminate symptoms. ${ }^{42} 4647$

So far most of the studies of PSS were based on clinical semiology. EEG was performed when indicated by clinically obvious seizures. This would miss many electrographic (subclinical/non-convulsive) seizures in patients with altered mental status. EEG is the best neurodiagnostic technique for detecting epileptic activity, especially in patients with non-convulsive PSS. EEG abnormalities in patients with stroke have been well described for many years and can be divided into three types: (1) non-specific abnormalities (diffused and focal polymorphic delta slowing, ipsilateral attenuation or loss of alpha and beta activities, as well as sleep spindle $)^{48}{ }^{49}$; (2) interictal epileptiform abnormalities which generally indicate an increased potential for developing seizures, like sharp and spike waves, lateralised periodic discharges (LPDs, formerly known as periodic lateralised epileptiform discharges) (figure 1), bilateral independent periodic discharges (BIPD formerly known as bilateral independent periodic lateralised epileptiform discharges) (figure 2), generalised periodic discharges (formerly known as generalised periodic epileptiform discharges), and temporal intermittent delta activity or lateralised rhythmic delta activity (figure 3$)^{750}$; and (3) ictal abnormalities which may present as rhythmic evolving theta, delta or alpha activities, rhythmic spike or spike waves, and electrodecremental activities. ${ }^{51-53}$ Mecarelli et $a t^{40}$ prospectively performed EEG on 232 patients with stroke, within 24 hours of admission, and found focal or diffused slowing in $84 \%$ of their patients, LPDs in $6 \%$ and other epileptiform abnormalities in $10 \%$ of their patients. Carrera $e$ t $a \tilde{l}^{33}$ studied 100 consecutive patients with acute stroke where cEEG was monitored. They found epileptiform abnormalities in 17\% (3\% LPDs and $14 \%$ other types of epileptiform discharges) and electrographic seizures in $11.7 \%$ of the cases. 


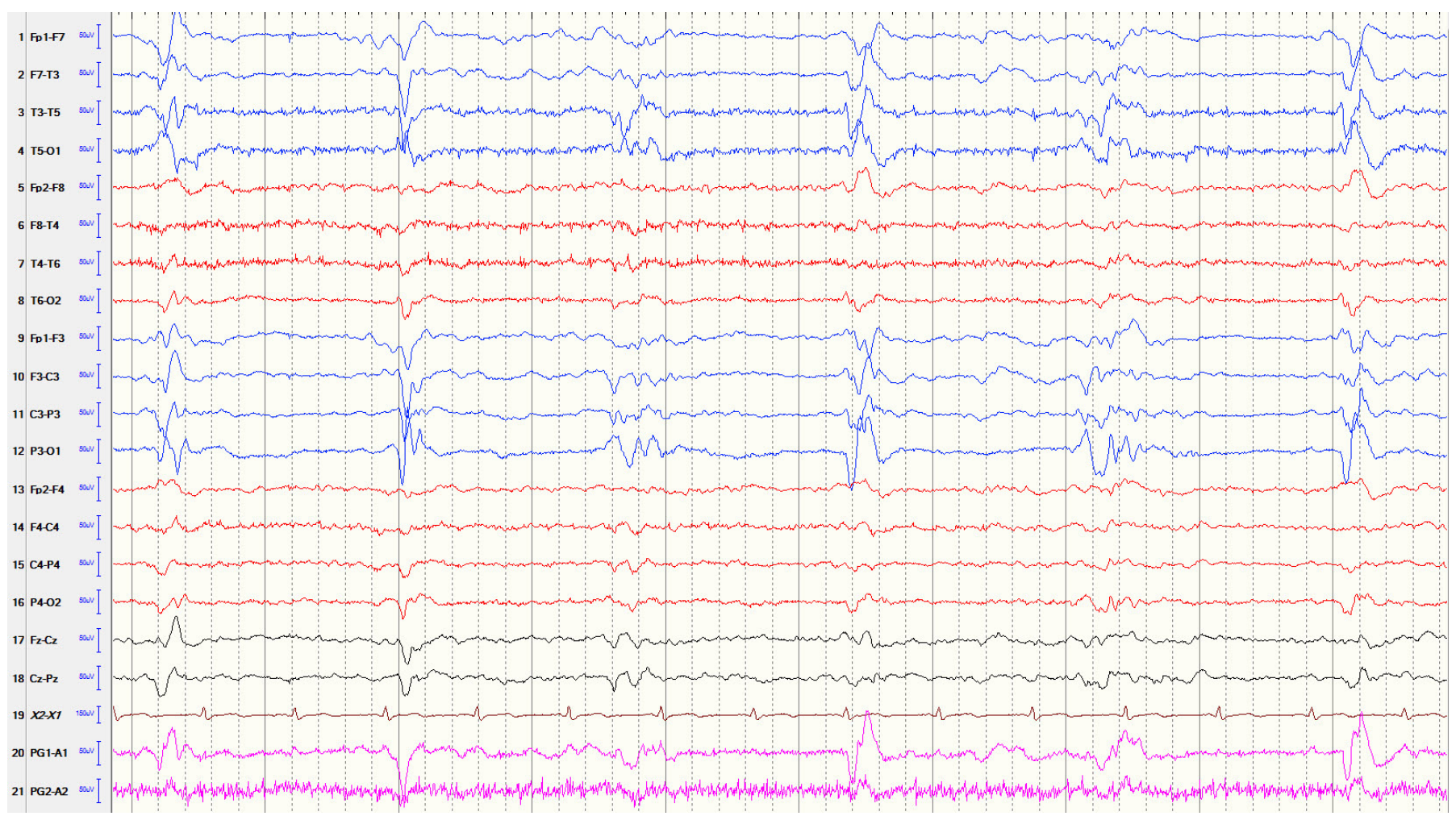

Figure 1 Lateralised periodic discharges (LPDs, formerly known as periodic lateralised epileptiform discharges). The EEG showed left side LPDs recurring at $0.5 \mathrm{~Hz}$ (discharges per second) with focal slowing in the same area. This EEG was obtained from a 61-year-old woman with left middle cerebral artery infarct and one-time generalised tonic-clonic seizure.

The value of interictal epileptiform abnormalities in predicting epileptic seizures or SE is still not clear. Interictal epileptiform abnormalities represent focal cortical irritation; among them, LPDs may be an expression of dynamic brain damage in the very acute stage and they increase the potential for developing seizures. In Mecarelli $e t a l$ s study, ${ }^{40}$ EEGs were performed within 24 hours of admission and the patients were followed up clinically for 1 week. The authors found that none of the patients with focal or diffused slowing developed epileptic seizures; $85.7 \%$ of patients with LPDs developed seizures, and $13 \%$ of patients with other epileptiform abnormalities had focal seizures. Among the patients with LPDs, $71 \%$ with seizure developed into SE, $90 \%$ convulsive SE and $10 \%$ none convulsive SE. Koren and colleagues ${ }^{52}$ in a small sample study found that in $23 \%$ of their patients, epileptiform discharges within the first $30 \mathrm{~min}$ of EEG developed into electrographic seizures, as shown in subsequent EEG. Multivariate analysis demonstrated that only early epileptiform discharge LPDs were independently associated with PSS. ${ }^{53}$

EEG can be performed as standard EEG (20-45 min), prolonged or extended EEG (from 1 hour to 2 hours), or cEEG (typically greater than 2 hours, in some studies 24 hours or greater). Previous studies have demonstrated that cEEG is superior to standard EEG in capturing interictal or ictal abnormalities, especially in cases of non-convulsive seizures and non-convulsive SE. ${ }^{72-54}$ However, there is no guideline regarding what type of EEG should be ordered in patients with stroke and the optimum time of continuous monitoring or recording seizure activity. Several studies in patients with acute stroke admitted to the stroke unit or ICU suggested cEEG should be performed on those patients with obvious clinical convulsive or rhythmic motor symptoms, and also on patients with a high NIHSS score at onset, coma or stupor, and intermittent altered mental status. ${ }^{78}{ }^{52-54}$ In 2015 the American Clinical Neurophysiology Society developed a consensus statement on cEEG in critically ill adults and children (box 1) which may also apply, at least partially, to patients with stroke. ${ }^{55}$

\section{TREATMENT OF PSS}

The treatment of PSS may be divided into two major categories: (1) prevention and prophylactic treatment and (2) symptomatic treatment. So far, data available to guide above issues are very sparse.

For PSS prevention and prophylactic treatment, although some studies advocated possible short-term prophylactic antiepileptic treatment for $\mathrm{ICH}^{5},{ }^{56}$ the American Heart Association and the European Stroke Organisation have stated that prophylactic administration of AEDs to prevent a seizure is not recommended for patients with stroke, including patients with $\mathrm{ICH}^{57-59}$ This is due to a lack of reliable randomised control trials which might prove that prophylaxis will prevent seizures. In addition, AEDs have non-negligible side 


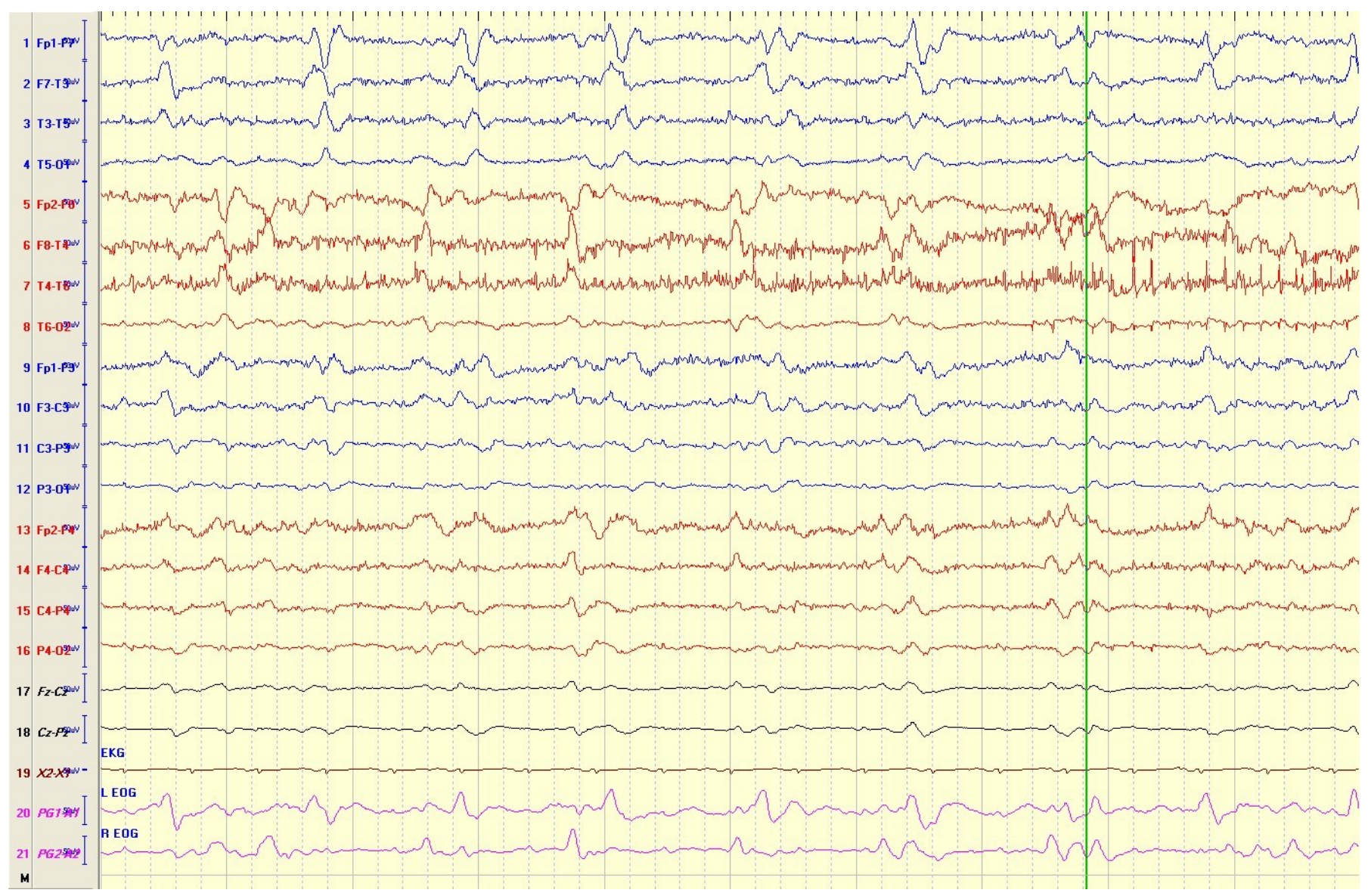

Figure 2 Bilateral independent periodic discharges (BIPDs, formerly known as bilateral independent periodic lateralised epileptiform discharges). The electroencephalogram (EEG) showed BIPDs are present over the bilateral temporal areas independently (unsynchronised). This EEG was obtained from a 34-year-old man with subarachnoid haemorrhage and several generalised tonic-clonic seizures.

effects, and some data also suggest that prophylactic use of AED therapy may be associated with poorer outcome. ${ }^{60}$ However, most of the prophylactic AED therapy trials were using older generation AEDs, and newer generation AEDs have fewer side effects and improved drug-todrug interaction profiles. Therefore, more prospective, randomised and controlled studies with newer generation AEDs need to be implemented in the future. Besides the traditional AEDs, several stroke preventive and other medications have been reported to have an effect on reducing the risk of PSS. Diuretics, for example, thiazides and furosemide, have been reported to show protective effects against seizures in animal models, and in observational epidemiological studies diuretics seem to reduce the risk of seizures in patients. ${ }^{56}$ Statin also has been studied for its antiepileptic effects. ${ }^{561-63}$ Guo et $a l^{62}$ found that statin use was associated with reduced risk of epilepsy among patients with ES after stroke. Etminan et $a t^{63}$ reported that statin use in patients with cardiovascular illness reduced the risk of subsequent hospitalisation for epilepsy. It has been suggested that statin antiepileptogenic effect may be related to its anticonvulsant potential, or anti-inflammatory effect or effect on brain blood barrier (BBB) injury. Theoretically, mitigation of the initial stroke effect to reduce brain damage should have an effect on preventing PSS or PSE. However, Tan $e t$ $a l^{64}$ retrospectively compared cases that were treated with tissue plasminogen activator (tPA) with cases that did not receive treatment, and showed that there was no significant difference in epilepsy incidence at 2 years $(10.8 \%$ with tPA vs $8.0 \%$ without tPA).

Regarding the symptomatic treatment of PSS, once a diagnosis of PSS is established, as a practical matter, the next important step is to decide whether immediate AED treatment should be initiated. It has been proposed, and is now a generally accepted principle, that when a patient presents with a second or more unprovoked recurrent seizures, an AED should be initiated because the risk for yet additional seizures is very high $(57 \%$ by 1 year and $73 \%$ by 4 years).$^{18}$ So by the definition, the PSE should always be treated with an AED. It also has been generally accepted that if a patient presenting with the first unprovoked seizure shows no significant high risk $(\geq 60 \%)$ for recurrent seizures, the AED treatment should be deferred until the recurrent seizure occurs. But for PSS, this concept cannot be applied because stroke produces a structural lesion, and the risk of recurrent seizures varies among different studies; therefore, immediate AED 


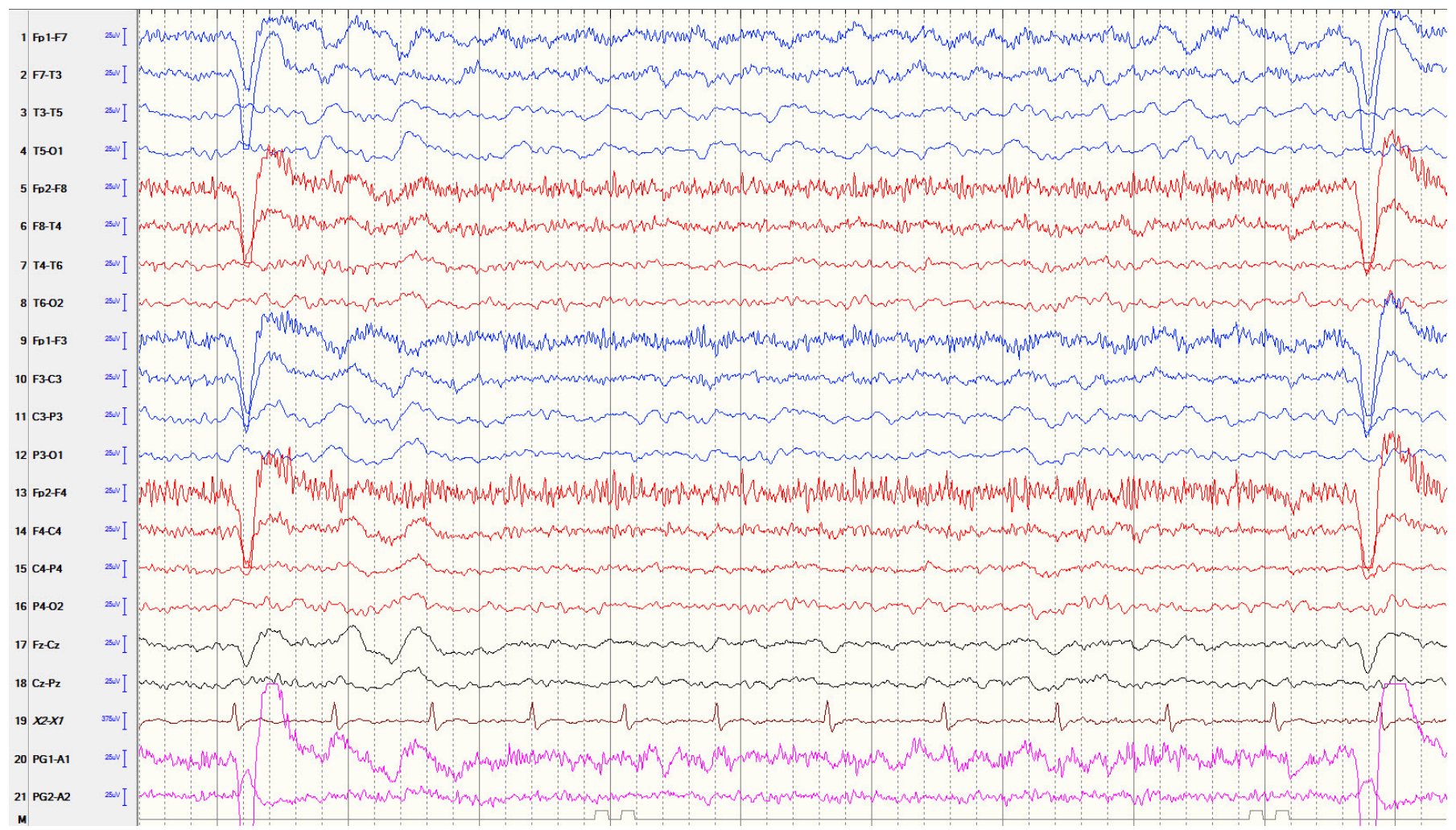

Figure 3 Lateralised intermittent rhythmic delta activity. The electroencephalogram (EEG) showed left side (mainly left temporal lobe) brief intermittent runs of rhythmic delta activity at $1-1.5 \mathrm{~Hz}$. This EEG was obtained from a $65-y e a r-o l d ~ w o m a n$ with 1-year history left side middle cerebral artery stroke and intermittent altered mental status and aphasia.

treatment is still under debate and depends on several clinical situations. $565765-68$

In PSS, ES may increase the risk of recurrent seizures, but the risk has been reported up to $33 \%$ and is not significantly higher than a single unprovoked seizure in cases with an aetiology other than stroke. Also, several studies consider that ES is a provoked seizure, so it does not typically warrant treatment with AEDs. ${ }^{22} 4256$ If multiple ES (within 24hours) occur or an ES occurs after ICH or haemorrhagic transformation, several studies suggest that a short-term treatment ( 1 month) may be beneficial

\section{Box 1 American Clinical Neurophysiology Society} consensus statement on continuous electroencephalogram (EEG) in critically ill adults and children (2015)

- Persistently abnormal mental status following generalised convulsive status epilepticus or other clinically evident seizures.

- Acute supratentorial brain injury with altered mental status.

- Fluctuating mental status or unexplained alteration of mental status without known acute brain injury.

- Generalised periodic discharges, lateralised periodic discharges or bilateral independent periodic discharges on routine or emergent EEG.

- Requirement for pharmacological paralysis and risk for seizures (eg, therapeutic hypothermia protocols, extracorporeal membrane oxygenation).

- Clinical paroxysmal events suspected to be seizures to determine whether they are ictal or non-ictal. for preventing LS. ${ }^{57586568}$ But the evidence is not strong enough to make a general recommendation.

In cases of LS, the risk of recurrent seizure is increased significantly. The risk has to be reported as high as 55\%-90\%. ${ }^{7131516}$ But the recurrence risk is not consistent among all studies. Some studies also reported that there is no difference on the recurrence risk between ES and LS. ${ }^{19-21}$ Regarding the AED treatment, one recent study prospectively reviewed 3792 patients with ischaemic stroke and identified 124 (3.3\%) patients with PSS. Among them, 48 cases were ES and 76 cases were LS. The authors observed AED treatment effects with a mean follow-up time of 29.9 months. In their patients with ES, 7 of 48 were not prescribed AEDs and the seizure recurrence rate was $43 \%$; 41 of 48 were treated with AEDs and the recurrence rate was $34 \%$. In their patients with LS, 7 of 76 were not prescribed AEDs and all of them (100\%) had recurrent seizures, and only $43 \%$ of patients with LS (69 of 76) treated with AEDs initially developed recurrent seizures. ${ }^{32}$ However, controversial studies also exist which indicate that immediate treatment after a first unprovoked seizure does not improve the remission rate. ${ }^{4575666}$ So the decision to initiate AED treatment after LS should be individualised, and primarily based on the characteristics of the seizure episode, the patient's preference and numerous other issues that could influence the patient's quality of life, for example, social and economic status, toleration of AED side effects, rehabilitation and functional recovery, comorbidities and drug-to-drug 
interactions with multiple medications, and so on. The risks and benefits of both options should be considered a discussion with the patient and his/her family. ${ }^{67}$

In cases of PSS with SE (includes subclinical or non-convulsive SE), the risk of recurrent seizures in 10 years was increased twofold to threefold and the severity of the seizures was also significantly increased ${ }^{67-69}$; therefore, a long-term antiepileptic treatment (similar to patients with recurrent unprovoked seizures) may be warranted. In patients with stroke with abnormal EEG, treatment should be considered based on the EEG pattern. Periodic discharge patterns, for example, LPDs and bilateral independent periodic discharges (BIPDs), are considered along a continuum between interictal and ictal state and may consequently induce neuronal injury, so long-term AED is recommended.

The principle of selecting an appropriate AED in patients with epilepsy has been well studied, which depends on numerous factors, such as seizure type/ epileptic syndrome, efficacy and adverse effects of respective AED, drug-to-drug interactions, age and gender, drug regimen, and so on. ${ }^{70}$ Nevertheless, the PSS typically occurred in the elderly population, and the stroke survivors have a tendency to present with other health-related issues, or treated with multiple medications and have alterations in drug metabolism, which make them more sensitive to adverse effects, so the selection of AEDs is more dependent on the potential side effects, tolerability, impact on the patient's rehabilitation and recovery, and drug-to-drug interactions. So far, the literature contains very limited data that specifically and prospectively evaluated antiepileptic therapy in PSS. However, taking into consideration the elderly population and underlying cerebral structural disturbance, conclusions derived from studies of AED treatment on focal (partial) seizures in the elderly population might be considered in the case of patients with PSS. The latest ILAE report recommends that for efficacy, as initial monotherapy in adults with focal seizures, carbamazepine (CBZ), levetiracetam (LEV), phenytoin (PHT) and zonisamide have level A evidence. For elderly patients, lamotrigine (LTG) and gabapentin (GBP) have level A evidence. ${ }^{71}$ In the Standard and New Antiepileptic Drugs (SANAD) trial, the efficacy and tolerance were compared among GBP, LTG, oxcarbazepine (OCBZ) and topiramate in patients with partial epilepsy. GBP was found to be less effective and LTG was better tolerated than the other drugs. ${ }^{72}$ In the Keppra vs Older Monotherapy in Epilepsy Trial (KOMET trial, LEV, valproic acid (VPA) and CBZ were compared as initial monotherapy in elderly patients (>60years old). It was found that LEV had better tolerance and less severe treatment withdrawal effects than other AEDs, but no difference was detected in drug efficacy. ${ }^{73}$ In a multicentre, double-blind, randomised trial study in elderly patients with focal epilepsy, Werhahn $e t a l^{74}$ found that LEV and LTG had better tolerability and less severe treatment withdrawal effects. Studies specifically targeted on PSS are typically of small sample, open-label or retrospect review, with low-level evidence. These studies also suggest that the newer generation AEDs LEV and LTG have better tolerability than the older generation AEDs, CBZ and PHT. ${ }^{75-78}$ Among the older generation AEDs, VPA seems to have better tolerance than CBZ and PHT. ${ }^{79}$

In general, based on currently available data, there was no significant difference in antiepileptic efficacy among most of the AEDs, regardless of whether the drugs are classified as newer or older generation AEDs, except for GBP which shows slightly lower efficacy. Of note, the newer generation AEDs has better side effect profile and less drug-to-drug interactions. LEV and LTG are the most studied newer generation AEDs and have the best drug tolerance. Drug side effects and drug-to-drug interactions profile in other newer generation AEDs, OCBZ, eslicarbazepine, lacosamide and perampanel, seems very encouraging as well, but their role in the treatment of PSS needs to be further studied. Another important issue in AED selection for PSS treatment is that it should be individualised as well, based on patients' comorbidities, other medications, social economic state and the AED side effect profile.

Regarding the response to AEDs, PSS and PSE have higher seizure control rate ( $\geq 65 \%$ seizure-free) compared with seizure control rate in patients with epilepsy in general ( $\leq 50 \%$ seizure-free)..$^{57-77}$ In a small sample study, Alvarez-Sabín $e t a l^{76}$ found that among 71 patients with LS treated with GBP, $81.7 \%$ achieved seizure-free status. But PSS and PSE have higher adverse events than other forms of seizures and epilepsy with an aetiology other than stroke. ${ }^{80-82}$ This may, at least partially, relate to the reduced hepatic clearance and renal elimination, and lower serum albumin level in elderly patients, which lead to increased plasma concentration of the AEDs. ${ }^{79}$

\section{CONCLUSION}

The management of PSS is a challenge due to lack of high-level evidence-based data and reliable guidelines. Neurologists and clinical practitioners who treat patients with stroke still face questions on a daily basis, such as how to make a correct diagnosis, the optimal time to start AED treatment and how to choose the right AED. This article reviewed recent advances in the literature and tried to address the above issues. Seizure is a clinical diagnosis which is based on clinical presentation in most instances, but PSS may have less significant clinical manifestations, consequently EEG; especially cEEG is a very important tool to establish correct diagnosis. So far, study data suggest most ES occurring after stroke are not warranted an AED treatment, due to lower seizure recurrence rate. Also adverse effects may outweigh treatment benefits, but there are some exceptions. For LS, the decision to initiate AED treatment should be individualised, primarily based on the characteristics of the seizure episode, the patient's preference and numerous other issues that could influence the patient's quality of life. So far, data suggest LEV and LTG are the best tolerated AEDs in PSS treatment. 
Contributors MYX is the sole author.

Funding The authors have not declared a specific grant for this research from any funding agency in the public, commercial or not-for-profit sectors.

Competing interests None declared.

Patient consent Not required.

Provenance and peer review Commissioned; externally peer reviewed.

Data sharing statement No additional data are available.

Open access This is an open access article distributed in accordance with the Creative Commons Attribution Non Commercial (CC BY-NC 4.0) license, which permits others to distribute, remix, adapt, build upon this work non-commercially, and license their derivative works on different terms, provided the original work is properly cited, appropriate credit is given, any changes made indicated, and the use is non-commercial. See: http://creativecommons.org/licenses/by-nc/4.0/.

\section{REFERENCES}

1. Benjamin EJ, Blaha MJ, Chiuve SE, et al. Heart disease and stroke statistics - 2017 update: A report from the american heart association. Circulation 2017;135:e229-e445.

2. Krueger H, Koot J, Hall RE, et al. Prevalence of individuals experiencing the effects of stroke in canada: Trends and projections. Stroke 2015;46:2226-31.

3. Hauser WA, Annegers JF, Kurland LT. Incidence of epilepsy and unprovoked seizures in Rochester, Minnesota: 1935-1984. Epilepsia 1993;34:453-8.

4. Tanaka T, Ihara M. Post-stroke epilepsy. Neurochem Int 2017;107:219-28.

5. Zelano J. Poststroke epilepsy: update and future directions. Ther Adv Neurol Disord 2016;9:424-35.

6. Pitkänen A, Roivainen R, Lukasiuk K. Development of epilepsy after ischaemic stroke. Lancet Neurol 2016;15:185-97.

7. Chung JM. Seizures in the acute stroke setting. Neurol Res 2014;36:403-6.

8. Reddy DS, Bhimani A, Kuruba R, et al. Prospects of modeling poststroke epileptogenesis. J Neurosci Res 2017;95:1000-16.

9. Myint PK, Staufenberg EF, Sabanathan K. Post-stroke seizure and post-stroke epilepsy. Postgrad Med J 2006;82:568-72.

10. Lynch MW, Rutecki PA, Sutula TP. The effects of seizures on the brain. Curr Opin Neurol 1996;9:97-102.

11. Camilo O, Goldstein LB. Seizures and epilepsy after ischemic stroke. Stroke 2004;35:1769-75.

12. Ferlazzo E, Gasparini S, Beghi E, et al. Epilepsy in cerebrovascular diseases: Review of experimental and clinical data with metaanalysis of risk factors. Epilepsia 2016;57:1205-14.

13. Stefanidou M, Das RR, Beiser AS, et al. Incidence of seizures following initial ischemic stroke in a community-based cohort: The Framingham Heart Study. Seizure 2017;47:105-10.

14. Arboix A, Comes E, García-Eroles L, et al. Prognostic value of very early seizures for in-hospital mortality in atherothrombotic infarction. Eur Neurol 2003;50 78-84.

15. So EL, Annegers JF, Hauser WA, et al. Population-based study of seizure disorders after cerebral infarction. Neurology 1996;46:350-5.

16. Beghi E, D'Alessandro R, Beretta S, et al. Incidence and predictors of acute symptomatic seizures after stroke. Neurology 2011;77:1785-93.

17. Lancman ME, Golimstok A, Norscini J, et al. Risk factors for developing seizures after a stroke. Epilepsia 1993;34:141-3.

18. Fisher RS, Acevedo C, Arzimanoglou A, et al. ILAE official report: a practical clinical definition of epilepsy. Epilepsia 2014;55:475-82.

19. Bryndziar T, Sedova P, Kramer NM, et al. Seizures following ischemic stroke: Frequency of occurrence and impact on outcome in a long-term population-based study. J Stroke Cerebrovasc Dis 2016;25:150-6.

20. Huang CW, Saposnik G, Fang J, et al. Influence of seizures on stroke outcomes: a large multicenter study. Neurology 2014;82:768-76.

21. Alvarez V. Acute seizures in the acute ischemic stroke setting: a step forward in their description. Neurology 2014;82:740-1.

22. Serafini A, Gigli GL, Gregoraci G, et al. Are Early Seizures Predictive of Epilepsy after a Stroke? Results of a Population-Based Study. Neuroepidemiology 2015;45:50-8.

23. Zhao $\mathrm{Y}$, Li X, Zhang $\mathrm{K}$, et al. The progress of epilepsy after stroke. Curr Neuropharmacol 2018;16:71-8.

24. Strzelczyk A, Haag A, Raupach $\mathrm{H}$, et al. Prospective evaluation of a post-stroke epilepsy risk scale. J Neurol 2010;257:1322-6.

25. Haapaniemi E, Strbian D, Rossi C, et al. The CAVE score for predicting late seizures after intracerebral hemorrhage. Stroke 2014;45:1971-6.
26. Zelano J, Redfors P, Åsberg S, et al. Association between poststroke epilepsy and death: A nationwide cohort study. Eur Stroke $J$ 2016;1:272-8.

27. Arntz RM, Rutten-Jacobs LC, Maaijwee NA, et al. Poststroke epilepsy is associated with a high mortality after a stroke at young age: Follow-up of transient ischemic attack and stroke patients and unelucidated risk factor evaluation study. Stroke 2015;46:2309-11

28. $\mathrm{Xu} \mathrm{T,} \mathrm{Ou} \mathrm{S,} \mathrm{Liu} \mathrm{X,} \mathrm{et} \mathrm{al.} \mathrm{Association} \mathrm{between} \mathrm{seizures} \mathrm{after} \mathrm{ischemic}$ stroke and stroke outcome: A systematic review and meta-analysis. Medicine 2016;95 e4117.

29. Laccheo I, Ablah E, Heinrichs R, et al. Assessment of quality of life among the elderly with epilepsy. Epilepsy Behav 2008;12:257-61.

30. Burneo JG, Fang J, Saposnik G. Investigators of the registry of the canadian stroke network. Impact of seizures on morbidity and mortality after stroke: A canadian multi-centre cohort study. Eur J Neurol 2010;17:52-8.

31. Lees A. Retrospective study of seizure-related injuries in older people: a 10-year observation. Epilepsy Behav 2010;19:441-4.

32. Kim HJ, Park KD, Choi KG, et al. Clinical predictors of seizure recurrence after the first post-ischemic stroke seizure. BMC Neurol 2016;16:212.

33. Koubeissi M. Seizures worsen stroke outcome: new evidence from a large sample. Epilepsy Curr 2015;15:30-1.

34. Dhanuka AK, Misra UK, Kalita J. Seizures after stroke : a prospective clinical study. Neurol India 2001;49:33-6.

35. Alberti A, Paciaroni M, Caso V, et al. Early seizures in patients with acute stroke: frequency, predictive factors, and effect on clinical outcome. Vasc Health Risk Manag 2008;4:715-20.

36. Fisher RS, Cross JH, D'Souza C, et al. Instruction manual for the ILAE 2017 operational classification of seizure types. Epilepsia 2017:58:531-42

37. Blum DE, Eskola J, Bortz JJ. Patient awareness of seizures Neurology. 1996;47:260-4

38. Claassen J, Mayer SA, Kowalski RG, et al. Detection of electrographic seizures with continuous EEG monitoring in critically ill patients. Neurology 2004;62:1743-8.

39. Maganti R, Gerber P, Drees C, et al. Nonconvulsive status epilepticus. Epilepsy Behav 2008;12:572-86.

40. Mecarelli O, Pro S, Randi F, et al. EEG patterns and epileptic seizures in acute phase stroke. Cerebrovasc Dis 2011;31:191-8.

41. Miyaji Y, Kawabata Y, Joki H, et al. Late Seizures after Stroke in Clinical Practice: The Prevalence of Non-convulsive Seizures. Intern Med 2017;56:627-30.

42. Kim BS, Sila C, et al. Seizures in Ischemic Stroke. In. Koubeissi MZ, ed. Seizures in cerebrovascular disorders. New York: Springer Science+Business Media, 2015

43. Saposnik G, Caplan LR. Convulsive-like movements in brainstem stroke. Arch Neurol 2001;58:654-7.

44. Ghika J, Bogousslavsky J, van Melle G, et al. Hyperkinetic motor behaviors contralateral to hemiplegia in acute stroke. Eur Neurol 1995;35:27-32

45. Kim JS. Involuntary movements after anterior cerebral artery territory infarction. Stroke 2001;32:258-61.

46. Siniscalchi A, Gallelli L, Malferrari G, et al. Limb-shaking transient ischemic attack associated with focal electroencephalography slowing: case report. J Vasc Interv Neurol 2012;5:3-5.

47. Knoflach M, Matosevic B, Meinhart M, et al. Prognostic relevance of limb shaking in symptomatic carotid artery occlusion. Cerebrovasc Dis 2011:32:35-40.

48. Jordan KG. Emergency EEG and continuous EEG monitoring in acute ischemic stroke. J Clin Neurophysiol 2004;21:341-52.

49. Macdonell RA, Donnan GA, Bladin PF, et al. The electroencephalogram and acute ischemic stroke. Distinguishing cortical from lacunar infarction. Arch Neurol 1988;45:520-4.

50. Reiher J, Beaudry M, Leduc CP. Temporal Intermittent Rhythmic Delta Activity (TIRDA) in the diagnosis of complex partial epilepsy: sensitivity, specificity and predictive value. Can J Neurol Sci 1989;16:398-401.

51. Fisher RS, Scharfman HE, deCurtis M. How can we identify ictal and interictal abnormal activity? Adv Exp Med Biol 2014;813:3-23.

52. Koren J, Herta J, Draschtak S, et al. Prediction of rhythmic and periodic EEG patterns and seizures on continuous EEG with early epileptiform discharges. Epilepsy Behav 2015;49:286-9.

53. Carrera E, Michel P, Despland PA, et al. Continuous assessment of electrical epileptic activity in acute stroke. Neurology 2006 67:99-104.

54. Claassen J, Jette N, Chum F, et al. Electrographic seizures and periodic discharges after intracerebral hemorrhage. Neurology 2007;69:1356-65. 
55. Herman ST, Abend NS, Bleck TP, et al. Consensus statement on continuous EEG in critically ill adults and children, part I: indications. J Clin Neurophysiol 2015;32:87-95.

56. Gilad R, Boaz M, Dabby R, et al. Are post intracerebral hemorrhage seizures prevented by anti-epileptic treatment? Epilepsy Res 2011;95:227-31

57. Lahti A-M, Saloheimo P, Huhtakangas J, et al. Poststroke epilepsy in long-term survivors of primary intracerebral hemorrhage. Neurology 2017;88:2169-75.

58. Hemphill JC III, Greenberg SM, Anderson CS, et al. Guidelines for the management of spontaneous intracerebral hemorrhage: A guideline for healthcare professionals from the American Heart Association/ American Stroke Association. Stroke 2015;46:2032-60.

59. Steiner T, Al-Shahi Salman R, Beer R, et al. European Stroke Organisation (ESO) guidelines for the management of spontaneous intracerebral hemorrhage. Int J Stroke 2014;9:840-55.

60. Winstein CJ, Stein J, Arena R, et al. Guidelines for adult stroke rehabilitation and recovery $\mathrm{A}$ guideline for healthcare professionals from the American Heart Association/American Stroke Association. Stroke 2016;47:e98-169.

61. Hesdorffer DC, Stables JP, Hauser WA, et al. Are certain diuretics also anticonvulsants? Ann Neurol 2001;50:458-62.

62. Guo J, Guo J, Li J, et al. Statin treatment reduces the risk of poststroke seizures. Neurology 2015;85:701-7.

63. Etminan M, Samii A, Brophy JM, et al. Statin use and risk of epilepsy: A nested case-control study. Neurology 2010;75:1496-500.

64. Tan M-L, Ng A, Pandher PS, et al. Tissue plasminogen activator does not alter development of acquired epilepsy. Epilepsia 2012;53:1998-2004.

65. Qian C, Löppönen P, Tetri S, et al. Immediate, early and late seizures after primary intracerebral hemorrhage. Epilepsy Res 2014:108:732-9.

66. Gilad R. Mnagement of seizures following a stroke: what are the options? Drugs Aging 2012;129:533-8.

67. Hesdorffer DC, Benn EKT, Cascino GD, et al. Is a first acute symptomatic seizure epilepsy? Mortality and risk for recurrent seizure. Epilepsia 2009:50:1102-8.

68. Tomari S, Tanaka T, Ihara M, Matsuki T, et al. Risk factors for post-stroke seizure recurrence after the first episode. Seizure 2017:52:22-6.

69. Rumbach L, Sablot D, Berger E, et al. Status epilepticus in stroke: Report on a hospital-based stroke cohort. Neurology 2000;54:350-4.
70. Sander JW. The use of antiepileptic drugs-principles and practice. Epilepsia 2004;45(s6):28-34.

71. Glauser T, Ben-Menachem E, Bourgeois B, et al. Updated ILAE evidence review of antiepileptic drug efficacy and effectiveness as initial monotherapy for epileptic seizures and syndromes. Epilepsia 2013;54:551-63.

72. Marson AG, Al-Kharusi AM, Alwaidh M, et al. The SANAD study of effectiveness of valproate, lamotrigine, or topiramate for generalised and unclassifiable epilepsy: an unblinded randomised controlled trial. The Lancet 2007;369:1016-26.

73. Pohlmann-Eden B, Marson AG, Noack-Rink M, et al. Comparative effectiveness of levetiracetam, valproate and carbamazepine among elderly patients with newly diagnosed epilepsy: subgroup analysis of the randomized, unblinded KOMET study. BMC Neurol 2016:16:149

74. Werhahn KJ, Trinka E, Dobesberger J, et al. A randomized, doubleblind comparison of antiepileptic drug treatment in the elderly with new-onset focal epilepsy. Epilepsia 2015;56:450-9.

75. Sykes L, Wood E, Kwan J, et al. Antiepileptic drugs for the primary and secondary prevention of seizures after stroke. Cochrane Database Syst Rev 2014;1:CD005398.

76. Alvarez-Sabin J, Montaner J, Padro L, et al. Gabapentin in late-onset poststroke seizures. Neurology 2002:59:1991-3.

77. Gilad R, Sadeh M, Rapoport A, et al. Monotherapy of lamotrigine versus carbamazepine in patients with poststroke seizure. Clin Neuropharmacol 2007;30:189-95.

78. Consoli D, Bosco D, Postorino P, et al. Levetiracetam versus carbamazepine in patients with late poststroke seizures: a multicenter prospective randomized open-label study (EpIC Project). Cerebrovasc Dis 2012;34:282-9.

79. Huang YH, Chi NF, Kuan YC, et al. Efficacy of phenytoin, valproic acid, carbamazepine and new antiepileptic drugs on control of late-onset post-stroke epilepsy in Taiwan. Eur J Neurol 2015;22:1459-68.

80. Ryvlin P, Montavont A, Nighoghossian N, et al. Optimizing therapy of seizures in stroke patients. Neurology 2006;67(12 Suppl 4):S3-S9.

81. Ramsay RE, Rowan AJ, Pryor FM. Special considerations in treating the elderly patient with epilepsy. Neurology 2004;62(5 Suppl 2):S24-S29.

82. Cloyd J, Hauser W, Towne A, et al. Epidemiological and medical aspects of epilepsy in the elderly. Epilepsy Res 2006;68(Suppl 1):39-48. 